The Astrophysical Journal, 623:L13-L16, 2005 April 10

(C) 2005. The American Astronomical Society. All rights reserved. Printed in U.S.A.

\title{
GALEX ULTRAVIOLET OBSERVATIONS OF THE INTERACTING GALAXY NGC 4438 IN THE VIRGO CLUSTER
}

\author{
A. Boselli,${ }^{1}$ S. Boissier,${ }^{2}$ L. Cortese, ${ }^{1}$ A. Gil de Paz,${ }^{2}$ V. Buat,${ }^{1}$ J. Iglesias-Paramo, ${ }^{1}$ B. F. Madore, ${ }^{2,3}$ T. Barlow, ${ }^{4}$ \\ L. Bianchi, ${ }^{5}$ Y.-I. Byun,${ }^{6}$ J. Donas, ${ }^{1}$ K. Forster,${ }^{4}$ P. G. Friedman, ${ }^{4}$ T. M. Heckman, ${ }^{7}$ P. Jelinsky, ${ }^{8}$ Y.-W. Lee, ${ }^{6}$ \\ R. Malina, ${ }^{1}$ D. C. Martin, ${ }^{4}$ B. Milliard, ${ }^{1}$ P. Morrissey, ${ }^{4}$ S. NefF, ${ }^{9}$ R. M. Rich, ${ }^{10}$ D. Schiminovich, ${ }^{4}$ M. Seibert,${ }^{4}$ \\ O. Siegmund ${ }^{8}$ T. Small, ${ }^{4}$ A. S. Szalay, ${ }^{7}$ B. Welsh, ${ }^{8}$ and T. K. Wyder ${ }^{4}$ \\ Received 2004 December 17; accepted 2005 February 3; published 2005 March 18
}

\begin{abstract}
We present GALEX near-ultraviolet $(2310 \AA)$ and far-ultraviolet $(1530 \AA)$ images of the interacting galaxy NGC 4438 (Arp 120) in the center of the Virgo Cluster. These images show an extended (20 kpc) tidal tail at the northwest edge of the galaxy that was previously undetected at other wavelengths; this tail is $15-25 \mathrm{kpc}$ from NGC 4438's nucleus. Except for in the nucleus, the UV morphology of NGC 4438 is totally different from the $\mathrm{H} \alpha+[\mathrm{N}$ II] morphology, which is more similar to the X-ray emission, confirming its gas cooling origin. We study the star formation history of NGC 4438 by combining spectrophotometric data in the UV-visible-nearIR wavelength range with population synthesis and galaxy evolution models. The data are consistent with a recent ( $10 \mathrm{Myr})$, instantaneous burst of star formation in the newly discovered UV northwestern tail that is significantly younger than the age of the tidal interaction with NGC 4435, dated by dynamical models at $\sim 100$ Myr ago. Recent star formation events are also present at the edge of the northern arm and in the southern tail, while totally lacking in the other regions, which are dominated by the old stellar population that was perturbed during the dynamical interaction with NGC 4435. The contribution of this recent starburst to the total galaxy stellar mass is lower than $0.1 \%$, an extremely low value for such a violent interaction. High-velocity, off-center tidal encounters such as that observed in Arp 120 are thus not sufficient to significantly increase the star formation activity of cluster galaxies.
\end{abstract}

Subject headings: galaxies: clusters: individual (Virgo) — galaxies: individual (NGC 4435, NGC 4438) — galaxies: interactions - ultraviolet: galaxies

\section{INTRODUCTION}

NGC 4438 (Arp 120) is the clearest example of an ongoing tidal interaction in a nearby cluster of galaxies. Apparently located close to the Virgo Cluster center ( $300 \mathrm{kpc}$ from M87), NGC 4438 is a bulge-dominated late-type spiral galaxy showing long tidal tails $(30 \mathrm{kpc})$ that are thought to be induced by a recent dynamical interaction with the nearby SB0 galaxy NGC 4435. Multifrequency observations covering the electromagnetic spectrum from X-rays (Kotanyi et al. 1983; Machacek et al. 2004) to radio continuum (Hummel \& Saikia 1991), including both spectrophotometric and kinematical (Kenney et al. 1995) data, have been carried out in the past to study the nature of this peculiar system. These observations have shown that the violent interaction between the two galaxies perturbed the atomic (Cayatte et al. 1990) and molecular (Combes et al. 1988) gas distribution, causing both gas infall toward the center,

\footnotetext{
${ }^{1}$ Laboratoire d'Astrophysique de Marseille, BP 8, Traverse du Siphon, F13376 Marseille, France.

${ }^{2}$ Observatories of the Carnegie Institution of Washington, 813 Santa Barbara Street, Pasadena, CA 91101.

${ }^{3}$ NASA/IPAC Extragalactic Database, California Institute of Technology, Mail Code 100-22, 770 South Wilson Avenue, Pasadena, CA 91125.

${ }^{4}$ California Institute of Technology, MC 405-47, 1200 East California Boulevard, Pasadena, CA 91125.

${ }^{5}$ Center for Astrophysical Sciences, Johns Hopkins University, 3400 North Charles Street, Baltimore, MD 21218.

${ }^{6}$ Center for Space Astrophysics, Yonsei University, Seoul 120-749, Korea.

${ }^{7}$ Department of Physics and Astronomy, Johns Hopkins University, Homewood Campus, Baltimore, MD 21218.

${ }^{8}$ Space Sciences Laboratory, University of California at Berkeley, 601 Campbell Hall, Berkeley, CA 94720.

${ }^{9}$ Laboratory for Astronomy and Solar Physics, NASA Goddard Space Flight Center, Greenbelt, MD 20771.

${ }^{10}$ Department of Physics and Astronomy, University of California, Los Angeles, CA 90095.
}

which might have induced nuclear activity (Kenney et al. 1995; Kenney \& Yale 2002; Machacek et al. 2004), and gas removal in the external parts, displacing part of the gas in the ridge in between the two galaxies (Combes et al. 1988).

Both multifrequency observational data (Kenney et al. 1995; Machacek et al. 2004) and model predictions (Combes et al. 1988; Vollmer et al. 2005) favor a recent ( 100 Myr) highvelocity, off-center collision between NGC 4435 and NGC 4438 .

Except for mild nuclear activity, it is still unclear whether or not the dynamical interaction between the two galaxies induced extranuclear star formation events: the low $\mathrm{H} \alpha /[\mathrm{N}$ II] ratio and the similar X-ray and $\mathrm{H} \alpha$ morphology of NGC 4438 indicate that the $\mathrm{H} \alpha$ emission in this case is not due to the ionizing radiation but is probably due to gas cooling phenomena (Machacek et al. 2004).

The UV emission (at $\sim 2000 \AA$ ) is dominated by young stars of intermediate masses $\left(2 M_{\odot}<M<5 M_{\odot}\right.$; e.g., Boselli et al. 2001) and provides us with an alternative star formation tracer. As part of the Nearby Galaxy Survey, we have observed the central $12 \mathrm{deg}^{2}$ of the Virgo Cluster using the Galaxy Evolution Explorer $(G A L E X)$. In this Letter we present the UV observations of the interacting galaxies NGC 4435 and NGC $4438 .{ }^{11}$

\section{DATA}

The GALEX data used in this work include far-ultraviolet (FUV; $\lambda_{\text {eff }}=1530 \AA, \Delta \lambda=400 \AA$ ) and near-ultraviolet (NUV; $\lambda_{\text {eff }}=2310 \AA, \Delta \lambda=1000 \AA$ ) images with a circular field of view of radius $\sim 0^{\circ}$. 6 . The spatial resolution is $\sim 5^{\prime \prime}$. Details of the GALEX instrument and data characteristics can be found in Mar-

${ }^{11}$ A distance of $17 \mathrm{Mpc}$ for Virgo is adopted. 


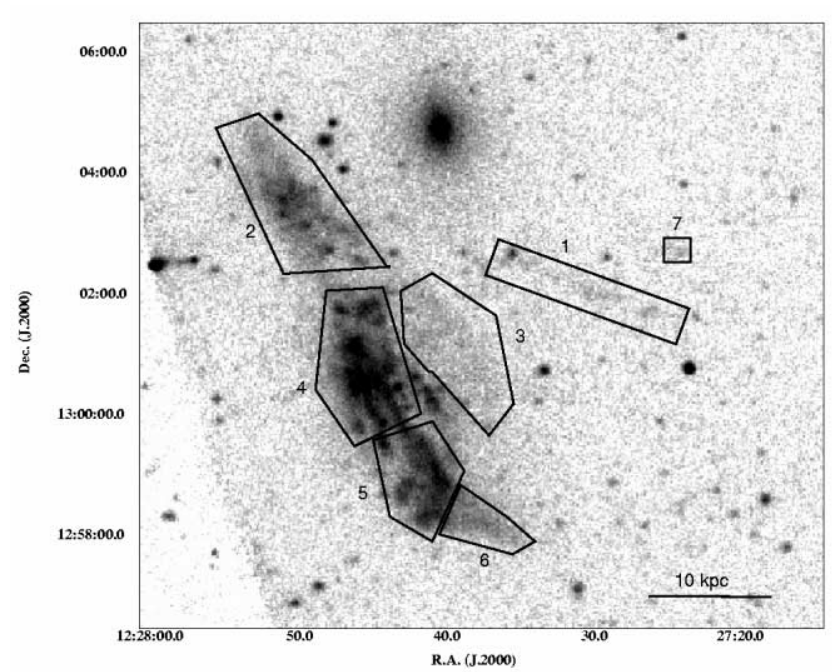

FIG. 1.-Combined NUV and FUV image of NGC 4438. The regions described in $\S 3$ of the text are labeled 1-7. The horizontal line is $10 \mathrm{kpc}$ long (assuming a distance of $17 \mathrm{Mpc}$ ).

tin et al. (2005) and Morrissey et al. (2005). The data (IR0.2 release) consist of two independent GALEX pointings centered at R.A.(J2000) $=12^{\mathrm{h}} 29^{\mathrm{m}} 01^{\mathrm{s}} 2$, decl. $(\mathrm{J} 2000)=+13^{\circ} 10^{\prime} 29^{\prime \prime} \cdot 6$ $\left(819\right.$ s) and R.A.(J2000) $=12^{\mathrm{h}} 25^{\mathrm{m}} 25^{\mathrm{s}} 2$, decl.(J2000) $=$ $13^{\circ} 10^{\prime} 29^{\prime \prime} 6(1511 \mathrm{~s})$, for a total of $2330 \mathrm{~s}$ of integration time.

To study the star formation history of NGC 4438, the UV data have been combined with visible and near-IR images of the galaxy taken from the GOLD Mine database (Gavazzi et al. 2003) ${ }^{12}$ from the Sloan Digital Sky Survey (SDSS) Third Data Release (Abazajian et al. 2005), from the Two Micron All Sky Survey (2MASS; Jarrett et al. 2003), and from the Canada-France-Hawaii Telescope (CFHT) and Subaru Telescope archives. These are $\mathrm{H} \alpha+[\mathrm{N}$ II] (Boselli \& Gavazzi 2002), $B$ (Boselli et al. 2003a), $K^{\prime}$ (Boselli et al. 1997), $u, g$, $r, i, z$ SDSS, $R$ CFHT and Subaru, and $H$ 2MASS images. For the main body of the galaxy (region 4 in Fig. 1; see next section) we added the integrated spectrum (3500-7000 $\AA$; Gavazzi et al. 2004). The current calibration errors of the NUV and FUV magnitudes are on the order of $\sim 10 \%$ (Morrissey et al. 2005), comparable to that at other frequencies.

\section{THE UV EMISSION AND THE STAR FORMATION HISTORY OF NGC 4438}

Figure 1 shows the UV image of NGC 4438, obtained by combining the NUV and FUV frames in order to increase the signal-to-noise ratio. The UV emission of the galaxy is mostly due to compact, bright regions in the central part of the galaxy (marked as region 4 in Fig. 1), in the northern tidal tail (region 2 ), and in the section of the southern tail closest to the main body of the galaxy (region 5). The UV emission is mostly diffuse in the extended western part of the northern tail (region 3) and at the edge of the southern tidal tail (region 6). Figure 1 shows the presence of extended and patchy emission to the northwest of the galaxy $(\sim 15-25 \mathrm{kpc}$ from the nucleus, marked as region $1)$. This feature, previously undetected in other visible and/or near-IR bands, is similar to a tidal tail $\sim 20 \mathrm{kpc}$ long and $\sim 2 \mathrm{kpc}$ wide. A similar smaller region $(\sim 2 \mathrm{kpc})$ at $\sim 25 \mathrm{kpc}$ from the nucleus is designated region 7 . The average NUV surface brightness of these features is $\sim 28.5 \mathrm{AB}$ mag $\operatorname{arcsec}^{-2}$, while they are

\footnotetext{
${ }^{12}$ See http://goldmine.mib.infn.it.
}

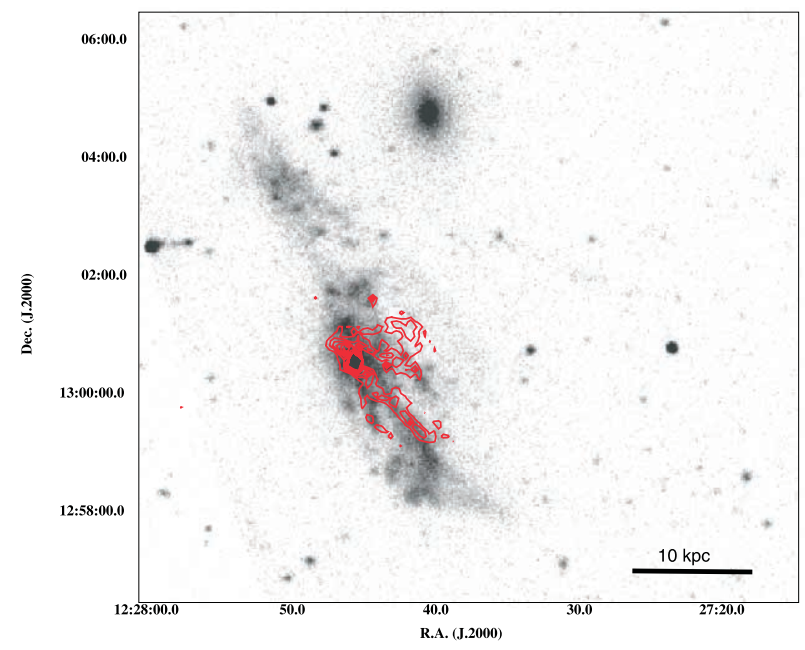

FIG. 2. $-\mathrm{H} \alpha+[\mathrm{N}$ II] contours (red, in arbitrary scale, in between $8 \times$ $10^{-17}$ and $6 \times 10^{-16} \mathrm{ergs} \mathrm{cm}^{-2} \mathrm{~s}^{-2} \operatorname{arcsec}^{-2}$, with $\sigma=5 \times 10^{-17} \mathrm{ergs} \mathrm{cm} \mathrm{cm}^{-2}$ $\mathrm{s}^{-2} \operatorname{arcsec}^{-2}$, from Boselli \& Gavazzi 2002) superposed to the NUV gray-level image of NGC 4438 .

undetected in the Subaru $R$-band (360 s) image down to a surface brightness limit of $\sim 27.8 \mathrm{mag} \operatorname{arcsec}^{-2}$.

The RGB image of the galaxy obtained by combining the FUV, $\mathrm{NUV}$, and $B$ frames, given in Figure 3 below, shows the color of the different regions: while the edge of both the northern and the southern tidal tails (regions 3 and 6) are red (thus dominated by relatively old stars), regions 2 and 5 , as well as the newly discovered regions 1 and 7, have blue colors and therefore seem to be dominated by a younger population. The $\mathrm{H} \alpha+[\mathrm{N}$ II] emission map, given in Figure 2 as a contour plot superposed on the NUV image of NGC 4438, shows a lack of massive, ionizing young OB $\operatorname{stars}^{13}$ (Kennicutt 1998) down to a surface brightness limit of $\sim 5 \times 10^{-17} \mathrm{ergs} \mathrm{s}^{-1} \mathrm{~cm}^{-2} \operatorname{arcsec}^{-2}$.

Extraplanar diffuse regions with an excess of the UV over $\mathrm{H} \alpha$ flux ratio (the same as that observed at $11 \mathrm{kpc}$ from the disk of M82) are often interpreted as being due to the UV radiation produced by the central starburst and locally scattered by diffuse dust (Hoopes et al. 2005). It is unlikely that scattered light is responsible for the UV emission in regions 2 and 5 since it comes from disk H II regions. The steep slope of the UV spectrum $(\beta=-2.32$ and -2.05 , as defined by Kong et al. 2004 for regions 1 and 7, respectively) is typical of a recent unreddened starburst (Calzetti 2001) and is unexpected in a scattering scenario since the dust albedo is greater in the NUV than in the FUV (Draine 2003). Furthermore, the lack of a powerful central starburst (as in M82) and the large distance of these relatively patchy regions from the nucleus seem to exclude the scattering scenario.

These data suggest that regions 1 and 7 (and to a lesser degree regions 2 and 5) are poststarbursts, induced by the violent interaction with NGC 4435, but that they lasted for a relatively short time, since they are not producing young, massive OB stars any more. This is probably because the atomic and molecular gases, which are needed to feed star formation,

\footnotetext{
${ }^{13}$ The $\mathrm{H} \alpha+[\mathrm{N}$ II] emission observed in region 5 has a different morphology than the UV emission: this evidence confirms the conclusions of Machacek et al. (2004) that the $\mathrm{H} \alpha+[\mathrm{N} \mathrm{II}]$ emission is not due to the ionizing radiation but is probably associated with the cooling gas.
} 


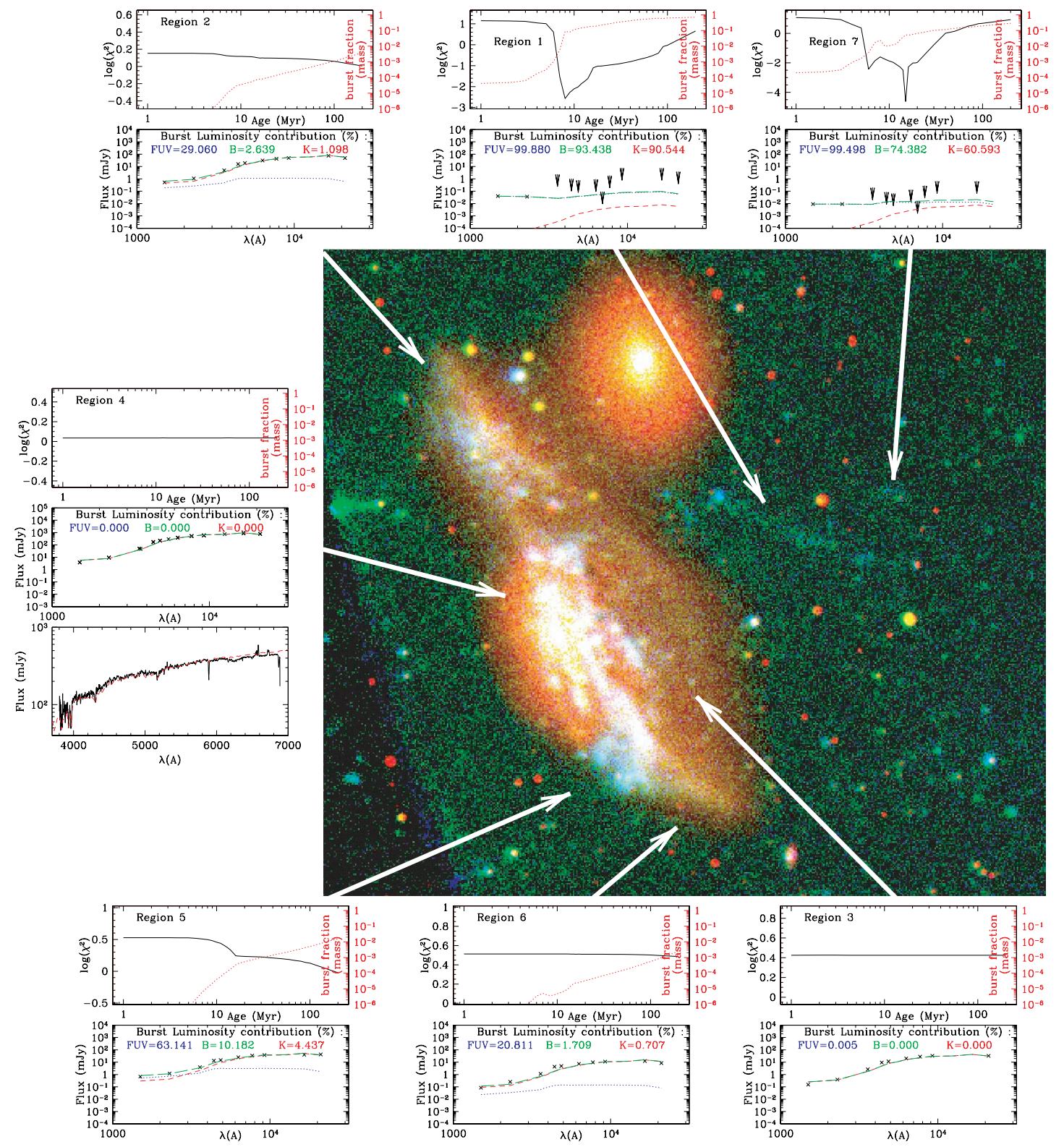

FIG. 3. - RGB (FUV $=$ blue, NUV $=$ green, $B=$ red) color map of NGC 4438 and NGC 4435. The SED of each region defined in Fig. 1 is given in the lower plot of each frame. Crosses indicate the observed data, arrows upper limits (in units of millijankys), the red dashed line the evolved population fit as determined by the model of Boissier \& Prantzos (2000), the dotted blue line the starburst SED (from Starburst 99), and the dashed green line the combined fitting model. The burst luminosity contribution (for the age corresponding to the minimum $\chi^{2}$ ) in the FUV, $B$, and $K$ is also given. The upper panel gives the variation of the reduced $\chi^{2}$ parameter (black continuum line, in logarithmic scale) and of the burst mass fraction (red dotted line) as a function of the age of the burst (in megayears). The lower panel of region 4 gives the integrated 3500-7000 $\AA, R=1000$ spectrum of the main body of the galaxy (black continuum line) compared to the fitted model (red dashed line).

have been removed during the interaction (Combes et al. 1988; Vollmer et al. 2005). ${ }^{14}$

In order to age-date the starburst and reconstruct the star formation history of the galaxy, we have determined the spectral energy distribution (SED) of these seven regions (see Fig. 3) and then fitted them with a model of galaxy evolution. To this end we make the assumption that dust attenuating the SED is present only in region 4, where we correct the UV-to-near-IR data using the far-IR-to-UV flux ratio as done in Boselli et al. (2003a). This restricted application is reasonable since no dust emission has been observed in the tidal tails with ISOCAM

\footnotetext{
${ }^{14}$ The upper limit of the $\mathrm{H}$ I surface density for these regions is $\sim 1 M_{\odot} \mathrm{pc}^{-2}$ (Cayatte et al. 1990).
}

(Boselli et al. 2003b); furthermore, in regions 1 and 7, dust is unexpected since it has not yet been produced by the young stellar population, as confirmed by the steep $\beta$-parameter.

We assume that NGC 4438 was a normal, late-type object before interacting with NGC 4435 . The models of the chemospectrophotometric evolution of the normal, spiral galaxies of Boissier \& Prantzos (2000), updated with an empirically determined star formation law (Boissier et al. 2003), are used to reconstruct the SED of the galaxy stellar population before the interaction. The two parameters of the model (spin $\lambda$ and rotational velocity $V$ ) are constrained by the total $H$-band luminosity and, to a minor extent, by the SED of the main body of the galaxy (region 4), composed of an old population with no sig- 
nificant contribution from the recent starburst (leading to $\lambda=$ 0.03 and $V=259 \mathrm{~km} \mathrm{~s}^{-1}$ ). We then assume that the underlying stellar population of each region, if present, is the one given by the model and removed from the main body of the galaxy by the tidal interaction, while the younger population is produced by the induced starburst. For each region, we combine the evolved stellar population with an instantaneous burst of star formation obtained using Starburst 99 (Leitherer et al. 1999) for a solar metallicity and a Salpeter initial mass function between 1 and $100 M_{\odot}$. For each age and intensity of the burst, we determine the best combination of evolved population + burst by fitting the FUV to the $K$-band SED and rejecting solutions that are in disagreement with the upper limits. We then adopt the age corresponding to the lowest reduced $\chi^{2} .{ }^{15}$ This exercise gives a consistent result: the strong UV emission of regions 1 and 7 is due to a coeval starburst $\sim 6-20$ Myr old (see region 1 in Fig. 3). The age and the duration of the starburst are strongly constrained by the lack of $\mathrm{H} \alpha$ emission and by the blue UV slope of the spectrum (lower limit to the age), and by the lack of an old stellar population (upper limit to the duration). Regions 2 and 5 are well fitted by an older starburst $(\geq 100 \mathrm{Myr})$ that ended $\sim 10$ Myr ago as indicated by the lack of any $\mathrm{H} \alpha$ emission and a redder UV slope $(\beta=-0.33$ and -0.67 in regions 2 and 5 , respectively). While the fraction of stars produced by this burst is dominant in regions 1 and 7, the sum of the stars produced by the burst in all regions (including the inner part) contributes to the total galaxy stellar mass by less than $0.1 \%$, an extremely low value for such a violent interaction.

\section{DISCUSSION AND CONCLUSION}

These observations have major consequences in constraining the evolution of cluster galaxies. A high-velocity off-center collision between two galaxies of relatively similar mass, whose violence is able to perturb the stellar distribution and thus produce important tidal tails, is insufficient to induce a

\footnotetext{
${ }^{15}$ All ages with $\chi^{2}<1$ are acceptable solutions. Given the small number of photometric points available for regions 1 and 7 (two GALEX bands), the fitted solution for a combination of a burst and an old population (two parameters) can be almost perfect (resulting in very low $\chi^{2}, \leq 10^{-2}$ ), as long as the obtained fit is in agreement with the limits at other wavelengths. Whenever the fit produces an SED that is not satisfying a detection limit, this solution is rejected.
}

significant instantaneous starburst. This result might be representative only of the nearby universe where encounters of gas-rich galaxies are probably rare since clusters are dominated by gas-poor early-type galaxies such as the companion galaxy NGC 4435. It is conceivable, however, that at higher redshifts, where clusters are forming, stellar masses produced by a starburst induced by interactions predicted by the models of Moore et al. (1996; galaxy harassment) might be more important given the higher fraction of gas-rich galaxies.

The other interesting result is the long time differential between the age of the interaction $(\sim 100 \mathrm{Myr}$ as determined by dynamical simulations; Combes et al. 1988; Vollmer et al. $2005)$ and the beginning of the starburst ( 10 Myr in regions 1 and 7, $100 \mathrm{Myr}$ in regions 2 and 5). This result is totally consistent with the models of Mihos et al. (1991) that predict for close-by encounters an enhancement of the star formation activity in the inner disk during some $100 \mathrm{Myr}$, stopping once the gas reservoir is exhausted as in NGC 4438. In the tidal tails, on the other hand, star formation is expected to increase after $\sim 100 \mathrm{Myr}$, which is the time needed by the gas to recollapse, but then to cease after a few megayears because the expansion of the tidal tail brings the gas surface density to subcritical values (no $\mathrm{H}$ I or $\mathrm{CO}$ has been detected in these regions). If these systems are dynamically stable and survive the interaction, they might be at the origin of some dwarf galaxies in the cluster that are similar to those observed in the Stephan's Quintet by Mendes de Oliveira et al. (2004) or in other interacting systems (Neff et al. 2005; Hibbard et al. 2005; Saviane et al. 2004). Being produced by a single starburst, these gas-poor systems might evolve into dwarf elliptical galaxies, which are typical of rich clusters; otherwise they will simply increase the fraction of unbound stars, thus contributing to the Virgo intracluster light (Willman et al. 2004).

GALEX (Galaxy Evolution Explorer) is a NASA Small Explorer, launched in 2003 April. We gratefully acknowledge NASA's support for the construction, operation, and science analysis of the GALEX mission, which was developed in cooperation with the Centre National d'Etudes Spatiales of France and the Korean Ministry of Science and Technology. We wish to thank the referee, V. Charmandaris, for his precious comments that helped improve the quality of the manuscript.

\section{REFERENCES}

Abazajian, K., et al. 2005, AJ, 129, 1755

Boissier, S., \& Prantzos, N. 2000, MNRAS, 312, 398

Boissier, S., Prantzos, N., Boselli, A., \& Gavazzi, G. 2003, MNRAS, 346, 1215

Boselli, A., \& Gavazzi, G. 2002, A\&A, 386, 124

Boselli, A., Gavazzi, G., Donas, J., \& Scodeggio, M. 2001, AJ, 121, 753

Boselli, A., Gavazzi, G., \& Sanvito, G. 2003a, A\&A, 402, 37

Boselli, A., Sauvage, M., Lequeux, J., Donati, A., \& Gavazzi, G. 2003b, A\&A, 406, 867

Boselli, A., Tuffs, R., Gavazzi, G., Hippelein, H., \& Pierini, D. 1997, A\&AS, 121, 507

Calzetti, D. 2001, PASP, 113, 1449

Cayatte, V., van Gorkom, J., Balkowski, C., \& Kotanyi, C. 1990, AJ, 100, 604

Combes, F., Dupraz, C., Casoli, F., \& Pagani, L. 1988, A\&A, 203, L9

Draine, B. T. 2003, ApJ, 598, 1017

Gavazzi, G., Boselli, A., Donati, A., Franzetti, P., \& Scodeggio, M. 2003, A\&A, 400, 451

Gavazzi, G., Zaccardo, A., Sanvito, G., Boselli, A., \& Bonfanti, C. 2004, A\&A, 417, 499

Jarrett, T., Chester, T., Cutri, R., Schneider, S., \& Huchra, J. 2003, AJ, 125, 525
Hibbard, J. E, et al. 2005, ApJ, 619, L87

Hoopes, C. G., et al. 2005, ApJ, 619, L99

Hummel, E., \& Saikia, D. 1991, A\&A, 249, 43

Kenney, J., Rubin, V., Planesas, P., \& Young, J. 1995, ApJ, 438, 135

Kenney, J., \& Yale, E. 2002, ApJ, 567, 865

Kennicutt, R., Jr. 1998, ARA\&A, 36, 189

Kong, X., Charlot, S., Brinchmann, J., \& Fall, S. 2004, MNRAS, 349, 769

Kotanyi, C., van Gorkom, J., \& Ekers, R. 1983, ApJ, 273, L7

Leitherer, C., et al. 1999, ApJS, 123, 3

Machacek, M., Jones, C., \& Forman, W. 2004, ApJ, 610, 183

Martin, D. C., et al. 2005, ApJ, 619, L1

Mendes de Oliveira, C., Cypriano, E. S., Sodré, L., Jr., \& Balkowski, C. 2004, ApJ, 605, L17

Mihos, C., Richstone, D., \& Bothun, G. 1991, ApJ, 377, 72

Moore, B., Katz, N., Lake, G., Dressler, A., \& Oemler, A. 1996, Nature, 379, 613

Morrissey, P., et al. 2005, ApJ, 619, L7

Neff, S. G., et al. 2005, ApJ, 619, L91

Saviane, I., Hibbard, J., \& Rich, M. 2004, AJ, 127, 660

Vollmer, B., Braine, J., Combes, F., \& Sofue, Y. 2005, A\&A, submitted

Willman, B., Governato, F., Wadsley, J., \& Quinn, T. 2004, MNRAS, 355, 159 